\title{
Amadeus
}

International Multidisciplinary Journal IISSN 2525-3281

DOI: 10.14295/aimj.v4i7.92

\section{Laparoscopy and Penetrating Abdominal Trauma: A Systematic Review}

Bráulio Filgueira Magalhães ${ }^{1}$; Pedro de Sousa Leiter,

Pedro Hugo Bezerra Maia

Filho $^{2}$;

George Wallisson Severo de

$\mathrm{Sa}^{4}$;

Whallyson Pinheiro Mascarenhas

Lucas Benevides Maia ${ }^{6}$,

Ticiane Ponciano de Oliveira

Lima $^{7}$;

Igor de Morais Marques ${ }^{8}$; Francisco Felippe de Araújo Rolim $^{9}$

\begin{abstract}
This article aims to evaluate the effectiveness and use of laparoscopy in penetrating abdominal trauma, thus providing a better definition of the role of laparoscopic approach in patients with PAD. A systematic review was performed by searching indexed articles in the Virtual Health Library and the Medical Literature Analysis and Retrieval System Online database (MEDLINE / PubMed), in which analysis after final analysis included 07 articles. In this study we identified seven articles that evaluated the use and contributions of the laparoscopic approach in patients with TAP, in which it was identified that the use as a diagnostic approach is widely used due to its benefits and safety. Regarding its therapeutic approach it was determined that in the largest of the studies there is indication, usefulness, reliability and accuracy of its use, however, for better results is essential experience of the surgical team in laparoscopic technique.
\end{abstract}

Keywords: Abdominal trauma; Laparoscopy and Penetrating Trauma; Abdominal injury; Laparoscopy; Penetrating wound.

\footnotetext{
${ }^{1}$ Residente Cirurgia Geral- Faculdade de Medicina Estácio de Juazeiro do Norte( FMJ), Juazeiro do Norte-CE, Brasil, braulio.magalhaes@ hotmail.com;

${ }^{2}$ Graduando em Medicina da Faculdade de Medicina Estácio de Juazeiro do Norte. Juazeiro do Norte, Ceará, Brasil; pedroed1913@hotmail.com;

${ }^{3}$ Residente Cirurgia Geral- Faculdade de Medicina Estácio de Juazeiro do Norte(FMJ), Juazeiro do Norte-CE, Brasil, pedrohugomaia@hotmail.com;

${ }^{4}$ Residente Cirurgia Geral- Hospital Regional do Cariri, Juazeiro do Norte-CE, Brasil, Georgewsevero@gmail.com.br;

${ }^{5}$ Residente Cirurgia Geral- Hospital Regional do Cariri, Juazeiro do Norte-CE, Brasil, Whallyson10@ hotmail.com;

${ }^{6}$ Residente Cirurgia Geral- Hospital Regional do Cariri, Juazeiro do Norte-CE, Brasil, benevides973@ hotmail.com;

${ }^{7}$ Residente Cirurgia Geral- Hospital Regional do Cariri, Juazeiro do Norte-CE, Brasil, tici_de_oliveira@hotmail.com;

${ }^{8}$ Residente Cirurgia Geral- Hospital Regional do Cariri, Juazeiro do Norte-CE, Brasil, Igormmarques@ hotmail.com. ${ }^{9}$ Professor MSc. Faculdade de Medicina Estácio de Juazeiro do Norte(FMJ), Juazeiro do Norte-CE, Brasil, felippe.rolim1@gmail.com
} 


\title{
Laparoscopia e Trauma Abdominal Penetrante: Uma Revisão Sistemática
}

\begin{abstract}
Resumo: O presente artigo tem como objetivo avaliar a eficácia e o uso da laparoscopia no trauma abdominal penetrante, para assim proporcionar uma melhor definição do papel da abordagem laparoscópica em pacientes com TAP. Foi realizada uma revisão sistemática através da busca de artigos indexados na Biblioteca Virtual em Saúde e na base de dados Medical Literature Analysis and Retrieval System Online (MEDLINE/PubMed), no qual a análise após analise final foram incluídos 07 artigos. Neste estudo foram identificados sete artigos que avaliaram o uso e as contribuições da abordagem laparoscópica em pacientes com TAP, no qual foi identificado que a utilização como abordagem diagnóstica é amplamente usada devido os seus benefícios e segurança. Em relação a sua abordagem terapêutica foi determinado que na maior dos estudos existe indicação, utilidade, confiabilidade e precisão do seu uso, entretanto, para melhores resultados é fundamental experiência da equipe cirúrgica na técnica laparoscópica.
\end{abstract}

Descritores: Traumatismo abdominal; Laparoscopia e Traumatismo penetrante; Abdominal injurie; Laparoscopy; Penetrating wound.

\section{Introdução}

Parajuli e outros colaboradores (2018) discorrem que em pacientes politraumatizado o trauma abdominal é considerado um dos principais agravos prevenis de morte. Em que a decisão de qual método ser realizado é fundamental para promover um melhor prognóstico do paciente. Neste contexto, a laparoscopia apresenta função primordial no tratamento de pacientes hemodinamicamente estáveis com possibilidade de lesão abdominal promovendo a diminuição de laparotomias não terapêuticas e, com isso reduzindo as complicações pós-operatórias.

Em pacientes com trauma abdominal penetrante (TAP) a laparoscopia diagnóstica (DL) é considerada uma abordagem essencial na avaliação das lesões, sendo que a maior parte dos pacientes atendidos são por arma branca casos (Koto; Matsevych; Aldous, 2018).

A taxa de lesões diafragmáticas não identificadas em pacientes assintomáticos que apresentam lesões toracoabdominais penetrantes é de aproximadamente $43 \%$. No qual a morbimortalidade devido à realização de laparotomias não terapêuticas é muito elevada e com isso cada vez mais se utiliza a laparoscopia diagnóstica que demonstra ser set eficiente 
e eficaz na avaliação desses pacientes. Vale salientar que nos últimos anos a intervenção terapêutica laparoscopia tornou-se uma das estratégias em pacientes estáveis com peritonite, com quadro de evisceração e ar intra-abdominal livre na avaliação préoperatória. Entretanto, o papel da laparoscopia com abordagem terapêutica não esta bem delimitada ainda (Koto; Mosai; Matsevych, 2017).

Chestovich e outros colaboradores (2015) descrevem que relativamente poucos estudos têm relatado sobre o uso terapêutico de laparoscopia trauma, sendo que a maioria descreve que existe certa limitação do seu uso na reparação diafragmática. Entretanto cada vez mais vem apresentando resultados significativos na reparação do fígado, estômago, intestino delgado, cólon, diafragma, da bexiga, e da parede abdominal; sendo assim considerada por alguns autores como método seguro e eficaz.

A laparoscopia é considerada como um dos principais métodos terapêuticos na abordagem eletiva que apresentam como benefícios à redução do quadro doloroso, diminuição do internamento hospitalar e recuperação funcional de forma mais ágil. Diante desse processo a cada dia vem sendo mais utilizada na emergência, principalmente em situações como apendicite, perfuração péptica e diverticulite. Quando se avalia seu uso no ambiente do trauma abdominal é notório sua segurança e eficácia como abordagem diagnóstica. Entretanto, depois de 20 anos de utilização como modalidade diagnóstica torna-se notório que maior parte dos cirurgiões resistem em utilizar a laparoscopia como abordagem terapêutica no trauma, no quais fatores relacionados como estabilidade hemodinâmica do paciente politraumatizado, eficácia e segurança são considerados componentes cruciais para essas escolhas (Chakravartty et al., 2017).

Neste contexto, nos indagamos: qual a eficácia e o uso da laparoscopia no trauma abdominal penetrante? É possível que a análise proposta pelo presente estudo possa proporcionar uma melhor definição do papel da abordagem laparoscópica em pacientes com TAP.

O objetivo geral deste trabalho foi realizar uma revisão sistemática da literatura, para avaliar a eficácia e o uso laparoscopia no trauma abdominal penetrante. Para isso, acreditou-se necessário também:

a) Avaliar criteriosamente aspectos relevantes em estudos randomizados, prospectivos e retrospectivos publicados entre 2014 e 2019 sobre os benefícios do da laparoscopia em paciente com trauma abdominal penetrante;

b) Realizar uma análise crítica dos dados identificados; 
c) Apresentar e discutir os resultados identificados a partir dos estudos.

\section{Materiais e Método}

Uma revisão sistemática apresenta como objetivo primordial realizar um processo de síntese das informações sobre o tema abordado com qualidade metodológica, com o propósito de evitar vieses em todas as etapas da pesquisa. Para tal, foram utilizados " $a$ priori" no protocolo do estudo, as seguintes etapas:
a) definição da pergunta de revisão;
b) critérios de inclusão e exclusão;
c) estratégias para a busca dos estudos;
d) seleção do material;
e) análise crítica dos estudos;
e) coleta e síntese dos dados.

\section{Definição da pergunta}

Quais os benefícios da laparoscopia em pacientes com trauma abdominal penetrante?

\section{Critérios de inclusão}

Consideraram-se elegíveis os estudos randomizados, prospectivos e retrospectivos publicados no período de janeiro de 2014 a novembro de 2019; em qualquer idioma e em revistas com revisão por pares, com fator de impacto e disponibilizados na íntegra. Além disso, os estudos deveriam descrever resultados de abordagens realizados em seres humanos e apresentassem uma amostra superior a 20 pacientes.

Foram incluídos todos os estudos que avaliaram a eficácia e os benéficos do laparoscopia em pacientes com trauma abdominal penetrante. Artigos que aparecerem em mais de um banco de dados serão incluídos apenas uma vez, com prioridade à base de dados SCOPUS. 


\section{Critérios de exclusão}

Foram excluídos relatos de caso, revisões, prefácios, comunicações breves e cartas ao editor, estudos com duplicata e/ou que apresentava erros sistemáticos, ambiguidade ou apresentação insuficiente dos resultados.

\section{Estratégia de busca e seleção do material}

Os estudos foram selecionados por meio de busca eletrônica nas bases de dados Medical Literature Analysis and Retrieval System Online (MEDLINE/PubMed) e Biblioteca Virtual em Saúde (BVS). Foi utilizado os descritores em Traumatismo abdominal, Laparoscopia e Traumatismo penetrante e os MeSH terms Abdominal injuries, Laparoscopy e Penetrating wound utilizando o operador Booleando AND com os descritores combinados dois a dois.

Os estudos potencialmente relevantes para serem incluídos na revisão sistemática foram obtidos pela leitura dos títulos, resumos e do texto integral sempre que o resumo não fosse esclarecedor relativamente aos critérios utilizados para a decisão de inclusão ou exclusão. Um só investigador foi responsável pela extração dos seguintes dados: nome dos autores, título do artigo e ano de publicação; tipo do estudo; tamanho da amostra estudada, país do estudo; periódico e desfecho.

Foi realizada de forma ativa a busca manual (handsearching) por literatura cinzenta (relatórios e/ou documentos governamentais, dissertações e/ou teses, revisões bibliográficas, anais científicos, livros com pertinência teórica na área), além da utilização de artigos de revisão não elegíveis segundo os critérios de busca para o estudo, a fim de serem referenciados no trabalho para se realizar um embasamento teórico sobre o tema.

\section{Resultados}

Os estudos incluídos foram realizados na África do Sul (três artigos), Inglaterra, Estados Unidos da América, Taiwan e Índia um estudo cada. Foi então produzida a Tabela 1 no qual descreve as características dos estudos que foram finalmente incluídos na revisão sistemática. 
Figura 1: Fluxograma da busca dos estudos

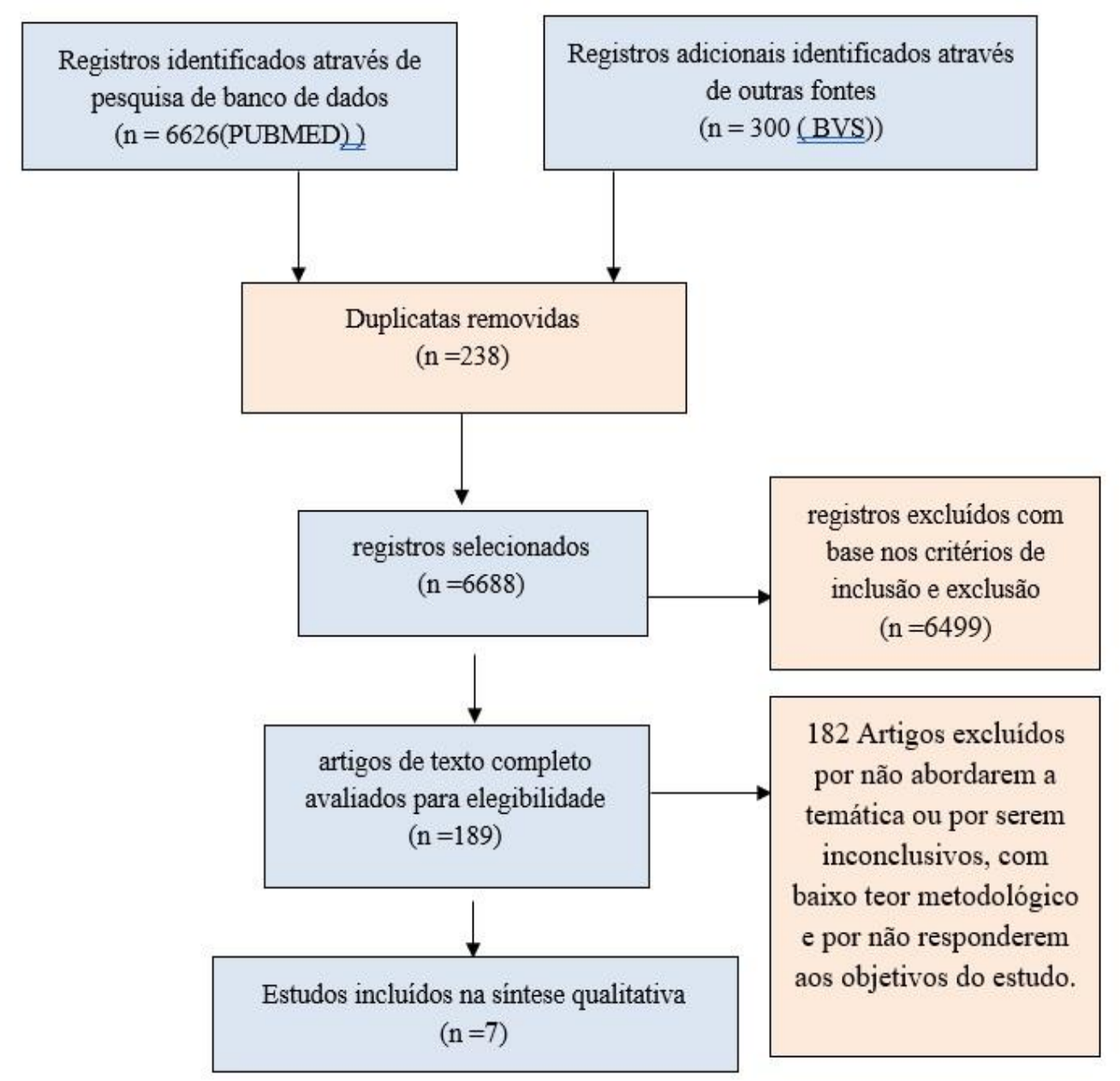

Fonte: elaborado pelos autores, (2019).

Na fase de identificação dos artigos, obtiveram-se 6926 registros, dos quais 238 foram excluídos por serem duplicatas. Além disso, 6499 foram excluídos após a análise dos critérios de inclusão e 182 por fugirem do tema, serem inconclusivos, com baixo teor metodológico e/ou por não responderem aos objetivos do estudo. Através da leitura detalhada dos estudos e pela confirmação de elegibilidade, 7 artigos foram inclusos como evidências. Foram identificados 6 estudos exclusivamente retrospectivos, enquanto que 01 foi retrospectivo e prospectivo. Na Tabela 2 é possível identificar os principais achados. 
Tabela 01: Características dos estudos que foram finalmente incluídos na revisão sistemática

\begin{tabular}{|c|c|c|c|c|c|c|}
\hline \multirow{2}{*}{ Autor/ Ano } & \multirow{2}{*}{$\begin{array}{l}\text { País de } \\
\text { Origem }\end{array}$} & \multirow{2}{*}{$\begin{array}{l}\text { Período do } \\
\text { estudo }\end{array}$} & \multirow{2}{*}{ Periódico } & \multirow{2}{*}{$\begin{array}{l}\text { Tipo de } \\
\text { Estudo }\end{array}$} & \multicolumn{2}{|c|}{$\begin{array}{c}\text { Tamanho da amostra } \\
\text { (n) }\end{array}$} \\
\hline & & & & & Laparoscopia & $\begin{array}{c}\text { Não } \\
\text { Laparoscopia }\end{array}$ \\
\hline $\begin{array}{l}\text { Chakravartty } \\
\text { et al., } 2017\end{array}$ & Inglaterra & $\begin{array}{c}\text { Janeiro de } \\
2004 \text { a } 2014\end{array}$ & $\begin{array}{c}\text { International } \\
\text { Journal of } \\
\text { Surgery } \\
\end{array}$ & $\begin{array}{l}\text { Estudo de } \\
\text { coorte } \\
\text { retrospectivo }\end{array}$ & 25 & 25 \\
\hline $\begin{array}{l}\text { Chestovich et } \\
\text { al., } 2015\end{array}$ & $\begin{array}{l}\text { Estados } \\
\text { Unidos da } \\
\text { América }\end{array}$ & $\begin{array}{c}\text { Janeiro de } \\
2008 \text { a } \\
\text { Dezembro de } \\
2013 \\
\end{array}$ & $\begin{array}{c}\text { Journal of } \\
\text { Trauma and } \\
\text { Acute Care } \\
\text { Surgery } \\
\end{array}$ & Retrospectivo & 138 & 380 \\
\hline $\begin{array}{c}\text { Koto; } \\
\text { Matsevych; } \\
\text { Aldous, } 2018\end{array}$ & $\begin{array}{l}\text { África do } \\
\quad \text { Sul }\end{array}$ & $\begin{array}{l}\text { Janeiro de } \\
2012 \text { a } \\
\text { Dezembro } \\
2015\end{array}$ & $\begin{array}{c}\text { Journal of } \\
\text { Laparoendoscopic } \\
\text { \& Advanced } \\
\text { Surgical } \\
\text { Techniques }\end{array}$ & Retrospectivo & 250 & 33 \\
\hline $\begin{array}{l}\text { Koto; Mosai; } \\
\text { Matsevych, } \\
2017\end{array}$ & $\begin{array}{l}\text { África do } \\
\quad \text { Sul }\end{array}$ & $\begin{array}{c}\text { Janeiro de } \\
2012 \text { a } \\
\text { Dezembro de } \\
2015\end{array}$ & $\begin{array}{l}\text { World Journal of } \\
\text { Emergency } \\
\text { Surgery }\end{array}$ & Retrospectivo & 83 & $\begin{array}{c}\text { Não } \\
\text { Informado }\end{array}$ \\
\hline $\begin{array}{l}\text { Lin; Chen; } \\
\text { Chen, } 2018\end{array}$ & Taiwan & $\begin{array}{c}\text { Janeiro de } \\
2006 \text { a } \\
\text { Dezembro de } \\
2015 \\
\end{array}$ & PloS one & Retrospectivo & 126 & 139 \\
\hline $\begin{array}{l}\text { Matsevych; } \\
\text { Koto; } \\
\text { Aldous, } 2017\end{array}$ & $\begin{array}{l}\text { África do } \\
\quad \text { Sul }\end{array}$ & $\begin{array}{c}\text { Janeiro de } \\
2012 \text { a } \\
\text { Dezembro de } \\
2015 \\
\end{array}$ & $\begin{array}{l}\text { International } \\
\text { Journal of } \\
\text { Surgery }\end{array}$ & Retrospectivo & 72 & $\begin{array}{l}\text { Não } \\
\text { informado }\end{array}$ \\
\hline $\begin{array}{l}\text { Parajuli et } \\
\text { al., } 2018\end{array}$ & Índia & $\begin{array}{c}\text { Janeiro de } \\
2008 \text { a Abril } \\
\text { de 2013; } \\
\text { Maio de } \\
2013 \text { a de } \\
\text { Março de } \\
2015\end{array}$ & $\begin{array}{c}\text { Surgical } \\
\text { laparoscopy, } \\
\text { endoscopy \& } \\
\text { percutaneous } \\
\text { techniques }\end{array}$ & $\begin{array}{l}\text { Retrospectivo } \\
\text { e Prospectivo }\end{array}$ & 119 & 1666 \\
\hline
\end{tabular}

Fonte: Elaborada pelos autores, (2019). 
Tabela 02: Principais achados

\begin{tabular}{|c|c|c|}
\hline TÍTULO & OBJETIVO & DESFECHO \\
\hline $\begin{array}{l}\text { Laparoscopy has a } \\
\text { therapeutic role in the } \\
\text { management of } \\
\text { abdominal trauma: a } \\
\text { matched-pair analysis. }\end{array}$ & $\begin{array}{l}\text { Comparar a laparoscopia com } \\
\text { a laparotomia no tratamento } \\
\text { de trauma abdominal em } \\
\text { pacientes hemodinamicamente } \\
\text { estáveis. }\end{array}$ & $\begin{array}{l}\text { Foi possível avaliar que a utilização da } \\
\text { laparoscopia como abordagem terapêutica em } \\
\text { pacientes com trauma abdominal é uma } \\
\text { ferramenta segura, no qual esta associada a uma } \\
\text { menor taxa de morbidade, diminuição de } \\
\text { medidas analgesias para o controle de dor, } \\
\text { como também taxa de internamento hospitalar } \\
\text { reduzido em comparação a laparotomia } \\
\text { terapêutica. Entretanto, vale ressaltar que o que } \\
\text { estes resultados dependem de fatores, como em } \\
\text { destaque as diferenças de técnicas utilizadas } \\
\text { pelos cirurgiões e sua experiência. }\end{array}$ \\
\hline $\begin{array}{l}\text { Minimally invasive is } \\
\text { maximally effective: } \\
\text { diagnostic and } \\
\text { therapeutic laparoscopy } \\
\text { for penetrating } \\
\text { abdominal injuries. }\end{array}$ & $\begin{array}{l}\text { Demonstrar o uso da } \\
\text { laparoscopia para diagnosticar } \\
\text { e tratar lesões penetrantes e } \\
\text { mostrar os benefícios de evitar } \\
\text { celiotomia em pacientes com } \\
\text { lesões passíveis de tratamento } \\
\text { laparoscópico }\end{array}$ & $\begin{array}{l}\text { Foi possível identificar uma menor taxa de } \\
\text { celiotomia em pacientes que foram submetidos } \\
\text { a laparoscopia em comparação aos que não } \\
\text { foram ( } 44 \text { vs } 380 \text { ). Além disso, pacientes } \\
\text { submetidos à laparoscopia como abordagem } \\
\text { terapêutica de lesões penetrantes apresentaram } \\
\text { como benefícios menor tempo de } \\
\text { hospitalização, menor taxa de infecção de } \\
\text { ferida no pós-operatório e complicações, como } \\
\text { íleo paralitico. Como abordagem diagnóstica é } \\
\text { um método seguro e eficaz que esta associada a } \\
\text { ausência de lesões perdidas. }\end{array}$ \\
\hline $\begin{array}{l}\text { Diagnostic laparoscopy } \\
\text { for trauma: How not to } \\
\text { miss injuries. }\end{array}$ & $\begin{array}{c}\text { Esclarecer a definiçãa de } \\
\text { laparoscopia diagnóstica (DL) } \\
\text { em cirurgia do trauma } \\
\text { abdominal penetrante (PAT). }\end{array}$ & $\begin{array}{l}\text { O estudo descreve que a DL é um procedimento } \\
\text { seguro e eficaz, no qual a realização de etapas } \\
\text { juntamente com um exame sistemático são } \\
\text { ferramentas cruciais para a diminuição de } \\
\text { lesões perdidas. Sendo a seleção do paciente o } \\
\text { passo inicial para as demais condutas. }\end{array}$ \\
\hline $\begin{array}{l}\text { The use of laparoscopy } \\
\text { in managing penetrating } \\
\text { thoracoabdominal } \\
\text { injuries in Africa: } 83 \\
\text { cases reviewed. }\end{array}$ & $\begin{array}{c}\text { Analisar a viabilidade e } \\
\text { segurança do uso da } \\
\text { laparoscopia no tratamento de } \\
\text { pacientes hemodinamicamente } \\
\text { estáveis com lesões } \\
\text { toracoabdominais penetrantes. }\end{array}$ & $\begin{array}{l}\text { Realizado retrospectivo com } 83 \text { pacientes no } \\
\text { qual identificou que a maior parte dos pacientes } \\
\text { apresentaram lesão diafragmática }(54 \%), 62 \% \\
\text { necessitaram de intervenção terapêutica e } \\
93,3 \% \text { foram tratados exclusivamente com a } \\
\text { laparoscopia. Por fim é notório a eficácia e } \\
\text { segurança na realização da técnica, como } \\
\text { também se pode afirmar que quadros de } \\
\text { peritonite em pacientes estáveis não } \\
\text { contraindica a laparoscopia. }\end{array}$ \\
\hline $\begin{array}{l}\text { Value of diagnostic and } \\
\text { therapeutic laparoscopy } \\
\text { for patients with blunt } \\
\text { abdominal trauma: A 10- } \\
\text { year medical center } \\
\text { experience. }\end{array}$ & $\begin{array}{c}\text { Avaliar se a laparoscopia } \\
\text { diagnóstica e terapêutica } \\
\text { apresenta a mesma eficácia de } \\
\text { pacientes com trauma } \\
\text { abdominal contuso em } \\
\text { comparação com o TAP. }\end{array}$ & $\begin{array}{l}\text { Foi realizado um estudo retrospectivo no qual } \\
\text { foi possível identificar uma taxa de sucesso de } \\
\text { laparoscopia terapêutica em } 92 \% \text { dos pacientes, } \\
\text { além disso, com menor taxa de quadros } \\
\text { hemorrágicos e complicações, sendo } \\
\text { considerada uma alternativa viável e segura. }\end{array}$ \\
\hline
\end{tabular}




\begin{tabular}{c|c|c}
\hline $\begin{array}{c}\text { Laparoscopic-assisted } \\
\text { approach for penetrating } \\
\text { abdominal trauma: A } \\
\begin{array}{c}\text { solution for multiple } \\
\text { bowel injuries. }\end{array}\end{array}$ & $\begin{array}{c}\text { Investigar o papel da } \\
\text { laparoscopia terapêtica no } \\
\text { tratamento de múltiplas lesões } \\
\text { intestinais e comparar a } \\
\text { abordagem laparoscópica } \\
\text { assistida(LAA) com a } \\
\text { laparoscopia terapêutica } \\
\text { realizada totalmente } \\
\text { laparoscopicamente (FTL). }\end{array}$ & $\begin{array}{l}\text { Foi identificado que a LAA é considerada uma } \\
\text { abordagem viável e segura na intervenção de } \\
\text { pacientes estáveis com PAT. Vale salientar que } \\
\text { seu uso é preferencial na terapêutica de } \\
\text { pacientes com múltiplas lesões intestinais, } \\
\text { proporcionando redução dos números de } \\
\text { conversão para laparotomia. }\end{array}$ \\
\hline $\begin{array}{c}\text { Role of laparoscopy in } \\
\text { patients with abdominal } \\
\text { trauma at level-I trauma } \\
\text { center. }\end{array}$ & $\begin{array}{c}\text { Avaliar a redução da } \\
\text { laparotomia não terapêutica } \\
\text { após o uso da laparoscopia, } \\
\text { como também a morbidade e o } \\
\text { tempo de internação. }\end{array}$ & $\begin{array}{c}\text { Foi realizado um estudo retrospectivo e } \\
\text { prospectivo com 3610 pacientes com trauma } \\
\text { promoveu redução das taxas de laparotomia não } \\
\text { terapêtica e apresentou menores complicações } \\
\text { e tempo de internamento. }\end{array}$ \\
\hline
\end{tabular}

Fonte: Elaborada pelos autores, (2019).

\section{Discussão}

Matsevych, Koto e Aldous (2017) descrevem que a principal etiologia responsável pelo quadro de TAP são ferimentos por arma branca (FAB) no qual promovem lesões principalmente da parte inferior do tórax e parede abdominal anterior.

Um estudo retrospectivo identificou que $83 \%$ dos pacientes submetidos a DL são decorrentes de facadas, com penetração da cavidade peritoneal ou retroperitoneal presente em 69\% dos casos (Koto; Matsevych; Aldous, 2018).

A realização da laparoscopia como abordagem diagnóstica no trauma após a introdução de técnicas e experiências aprimoradas proporcionou efeitos significativos no prognóstico do paciente, no qual apresenta taxas inferiores a $1 \%$ de lesões não identificadas, além disso, reduziu significativamente a realização da laparotomia negativa em aproximadamente 52\% (Chakravartty et al., 2017).

É notório que a laparoscopia é fundamental como abordagem diagnóstica de lesões intra-abdominais. Recentemente foram lançados diversos relatórios que descrevem a exploração e o uso da laparoscopia terapêutica de lesões traumáticas, entretanto existe uma hesitação para ser utilizada de maneira contundente no trauma. No qual os principais fatores que proporcionam essa hesitação são: relatos iniciais de lesões perdidas, incapacidade de visualização de todas as áreas do abdômen e tempo operatório superior a laparotomia. Entretanto, estudos recentes tem demostrado que existe eficácia terapêuticos superior a laparotomia no tratamento de lesões selecionadas, no entanto, carecem de mais evidencias cientificas. Os principais benefícios dos procedimentos laparoscópicos são 
tempo de permanência hospitalar inferior, quadro álgico menor no pós-operatório, incisões pequenas e retorno as atividades de forma mais precoce em comparação a procedimentos que necessitam de grandes incisões (Chestovich et al., 2015).

Neste estudo foram identificados sete artigos que avaliaram o uso e as contribuições da abordagem laparoscópica em pacientes com TAP, no qual foi identificado que a utilização como abordagem diagnóstica é amplamente usada devido os seus benefícios e segurança. Em relação a sua abordagem terapêutica foi determinado que na maior dos estudos existe indicação, utilidade, confiabilidade e precisão do seu uso, entretanto, para melhores resultados é fundamental experiência da equipe cirúrgica na técnica laparoscópica. Portanto pode-se afirmar que a laparoscopia pode usada na triagem, no diagnóstico e intervenção terapêutica dos casos de TAP e com isso proporcionando redução do internamento hospitalar, do uso de medicações para controle álgico, diminuição de hemorragias e complicações intra e pós-operatórias, como também redução da taxa de laparotomias não terapêuticas.

A laparoscopia diagnóstica (DL) é considerada uma abordagem aceita na avaliação de pacientes com TAP, apresentando uma sensibilidade, especificidade e precisão de aproximadamente $100 \%$ dos casos. $\mathrm{Na}$ abordagem do trauma é frequente a conversão de DL para laparotomia, principalmente na presença de múltiplas lesões intestinais, entretanto cada vez mais esta sendo implantada a abordagem laparoscópica assistida (LAA). Em um estudo realizado com 72 pacientes com PAT submetidos à abordagem terapêutica laparoscópica foi identificado que 65 pacientes foram tratados com a LAA sem necessidade de laparotomia (Matsevych; Koto; Aldous, 2017).

Além disso, Chestovich e outros colaboradores (2015) realizaram um estudo retrospectivo com 539 pacientes atendidos em um centro de trauma decorrente de lesões penetrantes avaliou a quantidade de pacientes que necessitaram de realização de celiotomia. No qual identificou que $73 \%$ dos pacientes foram submetidos à celiotomia de forma imediata, enquanto que $27 \%$ foram submetidos à laparoscopia inicial. Dos pacientes que passaram por laparoscopia, 24 foram convertidos a celiotomia decorrentes de lesões não passíveis de reparação laparoscópica, violação peritoneal, preferência cirurgião, hemoperitônio e instabilidade hemodinâmica. Portanto é notório que o uso da laparoscopia diagnóstica diminui a taxa de celiostomias desnecessárias, principalmente em pacientes com lesões penetrantes. 
Corroborando com o autor supracitado a laparoscopia diagnóstica (DL) foi inicialmente utilizada como medida de triagem, entretanto nos últimos anos devido à capacidade de avaliar a cavidade peritoneal de uma forma completa e sistemática tornou-se abordagem essencial no diagnóstico de lesões penetrantes abdominais. Vale ressaltar que cada vez mais é utilizada como intervenção terapêutica, sendo considerada viável e segura com taxa de realização superior a 55\% dos casos. Um estudo realizado com 250 pacientes submetidos a procedimentos laparoscópicos foi identificado que $45 \%$ foram submetidos à abordagem diagnóstica e 55\% a procedimento terapêutico com uma taxa de conversão para laparotomia de 1,7\% decorrente de quadros hemorrágicos contínuos, instabilidade do paciente, baixa visibilidade e falha dos equipamentos. Os autores afirmam que todo paciente com PAT estável deve ser abordado por laparoscopia devida apresentar inúmeros benefícios (Koto; Matsevych; Aldous, 2018).

Em um estudo retrospectivo foi avaliado pacientes admitidos em um centro de trauma e verificou-se que a laparoscopia foi implementada ao algoritmo de abordagem terapêutica de pacientes hemodinamicamente estáveis com facadas abdominais desde o ano de 2003. Além disso, torna-se notório que para melhores resultados é fundamental a presença da experiência adequada no manejo de procedimentos laparoscópicos na abordagem de pacientes com PAT (Lin; Chen; Chen, 2018).

Em pacientes com trauma abdominal penetrante (TAP) foi identificado que a laparoscopia proporcionou a diminuição de laparotomias não terapêuticas, como também promoveu a redução do tempo de permanência hospitalar, entretanto existem controvérsias em relação a sua eficácia terapêutica, principalmente relacionado a possibilidade de lesões não identificadas. Vale salientar que como medida diagnóstica ela é considerada como ferramenta precisa em pacientes com TAP e com isso sendo cada vez mais usada (Lin; Chen; Chen, 2018).

Um estudo realizado com 3610 pacientes com trauma abdominal a laparoscopia diagnóstica foi realizada em 119 pacientes no qual foi identificado uma taxa de redução da laparotomia não terapêutica em 55,4\% dos casos. Além disso, no estudo a laparoscopia apresentou uma precisão de $100 \%$ na identificação das lesões. Em relação a seu uso como abordagem terapêutica foi constatada um decrescimento do quadro de infecção e sepse, como também redução do tempo de internamento hospitalar (Parajuli et al., 2018).

Koto, Mosai e Matsevych (2017) realizaram um estudo com 83 pacientes estáveis que foram submetidos a procedimentos laparoscópicos decorrente de lesões 
toracoabdominais penetrantes no qual $62 \%$ foram realizaram intervenção terapêutica. No qual foi identificado uma taxa de complicações operatórias de $16 \%$ e intraoperatórias de $2 \%$. No total foi identificada uma de taxa de sucesso de $93 \%$ no uso exclusivo de laparoscopia e $82 \%$ dos pacientes não tiveram nenhuma intercorrência e nenhuma lesão foi perdida. Portanto, pode-se afirmar que existem viabilidade e confiança na realização da laparoscopia terapêutica nesses pacientes.

Ao comparar métodos laparoscópicos na abordagem de pacientes com TAP foi identificado que a LAA é considerada de escolha em pacientes com múltiplas lesões e/ou situações de maior gravidade no intestino, enquanto que a FTL é reservado na abordagem de lesões intestinais isoladas e outras lesões de órgãos intra-abdominais (Matsevych; Koto; Aldous, 2017).

Chakravartty e outros colaboradores (2017) relatam em seu estudo que apesar da TL ter apresentado um número menor de complicações pós-operatórias em comparação ao grupo que realizou laparotomia, existir segurança e viabilidade para realização do procedimento, deve-se avaliar a segurança do paciente e ponderar na escolha do método. Deve-se ter em mente que em pacientes hemodinamicamente instáveis a realização da TC é contraindicação absoluta. Além disso, em pacientes com lesão cerebral traumática grave o aumento a pressão intra-abdominal pode promover o aumento da pressão intracerebral e promover mais danos cerebrais, com isso, seu uso não é indicado.

Vale salientar que a experiência do cirurgião, alinhada a uma sistematização das técnicas laparoscópicas são fundamentais para a identificação de melhores resultados. Além disso, pode ser utilizada em várias abordagens.

Matsevych, Koto e Aldous (2017) abordam que alguns estudos relatam que quadros de evisceração são considerados como contraindicação para abordagem terapêutica laparoscópica, entretanto em seu estudo retrospectivo foi identificado que 25 pacientes que apresentaram evisceração foram abordados com a LAA, sendo considerada viável e segura.

Chakravartty e outros colaboradores (2017) descrevem em seu estudo que a maior parte dos pacientes com trauma abdominal que foram submetidos à TL foram decorrentes lesões de penetrante $(n=21)$, entre elas umas com evisceração do intestino delgado devido uma facada e uma lesão por arma de fogo. É importante compreender que as principais lesões que são reparadas com o uso da TL foram o diafragma (54\%), posteriormente omento / mesentério (13\%), fígado (13\%), estômago (7\%), intestino delgado (5\%), cólon $(3 \%)$ e baço $(3 \%)$. Salienta-se que lesões retroperitoneais necessitam de inspeções mais 
detalhadas, e com isso a depender da situação é necessário à realização da lapatoromia para avaliação da necessidade de intervenções adicionais. A utilização de manobras que proporcionem melhores resultados na TL são fundamentais, como por exemplo, a inclinação da mesa que possibilita ao cirurgião a realização de manobras laparoscópicas, principalmente ao mobilizar o intestino com o objetivo de promover uma lavagem ou sucção, além disso é fundamental a realização da sutura intra-corporal, particularmente em lesões de intestino delgado, estomago e diafragma.

Koto, Matsevych e Aldous (2018) descrevem que para melhores resultados na realização da laparoscopia diagnóstica torna-se essencial a realização de etapas prédeterminadas do procedimento e um exame laparoscópico sistemático e com isso promove uma diminuição significativa de lesões perdidas. No diagnóstico de lesões por trauma abdominal a avaliação deve seguir a seguinte ordem: diafragma, fígado e vesícula biliar, baço, parede anterior do estômago, pâncreas e áreas afins retroperitoneais, duodeno acima do mesocólon, duodeno abaixo mesocólon, intestino delgado executado a partir do ligamento de Treitz até a junção ileocecal, cólon direito, cólon transverso, cólon esquerdo, cavidade pélvica (o reto e a bexiga urinária), as áreas adicionais de interesse e outras áreas que exigem atenção extra (por exemplo, a avaliação da aorta) e por ultimo a decisão de prosseguir com a laparoscopia terapêutica ou conversão para laparotomia. A realização deste modelo sistemático promoveu a redução da taxa de lesões perdidas de $13 \%$ para abaixo de $1 \%$, entretanto, esse processo de exploração laparoscópica depende de cada centro.

É notório que pacientes com TAP com múltiplas lesões intestinais quando submetidos a laparotomia apresentam taxas de morbimortalidade elevadas, sendo $21,6 \%$ a de morbidade e 3,6 a de mortalidade. Em pacientes com abordagem laparoscópica foi identificado uma morbidade de $12,5 \%$ na terapia realizada totalmente laparoscopicamente (FTL) e $17 \%$ na LAA; quando avaliado a mortalidade identificou-se valores de $1,4 \%$ e $1,5 \%$, respectivamente. Portanto é visível que a abordagem laparoscópica nesses pacientes apresenta uma morbimortalidade significativamente inferior a pacientes submetidos à laparotomia. Entretanto para a reprodução de dados semelhantes torna-se fundamental a experiência dos profissionais na cirúrgica laparoscópica (Matsevych; Koto; Aldous, 2017).

Chakravartty e outros colaboradores (2017) realizaram um estudo retrospectivo com pacientes submetidos à cirurgia por trauma abdominal entre janeiro de 2004 e 2014, comparando os resultados de 25 pacientes submetidos à laparoscopia terapêutica (TL) com 
25 submetidos à laparotomia terapêutica (LT) no qual afirmam que a TL apresenta um tempo de procedimento semelhante com a LT, com baixa taxa de conversão $(1 / 25)$, com menor morbidade (P 0,02), com benefícios significativos na diminuição do uso de opióides $(\mathrm{P}=0,002)$ e tempo de internação hospitalar $(\mathrm{P}=0,03)$. Portanto, o uso da TL em pacientes com trauma abdominal pode ser considerada uma ferramenta segura em pacientes estáveis. Vale ressaltar que o estudo em questão apresentava limitações, entre elas o tipos de procedimentos realizados em cada grupo não serem exatamente idênticos e a questão da decisão entre a LT e a TL ser decorrente da experiência do cirurgião em operação.

\section{Considerações Finais}

Portanto a revisão sistemática foi composta por sete artigos que avaliaram a eficácia e o uso da laparoscopia em paciente com TAP. Esses estudos sugerem que a laparoscopia apresenta um papel fundamental na abordagem desses pacientes, sendo a experiência dos cirurgiões essenciais para promoção de melhores resultados no prognóstico dos pacientes, principalmente em pacientes com lesão diafragmática, do abdômen anterior e intestinal. Além disso, é notório a sua eficácia como abordagem diagnóstica e apresenta um elevado potencial como método terapêutico.

Vale salientar que os estudos sobre o tema, principalmente em relação a sua abordagem terapêutica são recentes, sendo a extensa realizada num período de dez anos, no estudo é notório que existem resultados significativos na redução do tempo de internamento, complicações e morbidades associadas. Além disso, na abordagem do trauma é considerada um método seguro e viável como método diagnóstico.

A falha metodológica mais identificada nos estudos foi à ausência da formação de dois grupo-controle em um dos estudos analisados e a quantidade pequena de artigos que abordem a laparoscopia como abordagem terapêutica de pacientes com TAP.

\section{Referências}

Chakravartty, Saurav et al. (2017). Laparoscopy has a therapeutic role in the management of abdominal trauma: a matched-pair analysis. International Journal of Surgery, v. 44, p. $21-25$. 
Chestovich, Paul J. et al. (2015). Minimally invasive is maximally effective: diagnostic and therapeutic laparoscopy for penetrating abdominal injuries. Journal of Trauma and Acute Care Surgery, v. 78, n. 6, p. 1076-1085.

Koto, Modise Z.; Matsevych, Oleh Y.; Aldous, Colleen. (2018). Diagnostic laparoscopy for trauma: How not to miss injuries. Journal of Laparoendoscopic \& Advanced Surgical Techniques, v. 28, n. 5, p. 506-513.

Koto, Zach M.; Mosai, Fusi; Matsevych, Y. (2017). The use of laparoscopy in managing penetrating thoracoabdominal injuries in Africa: 83 cases reviewed. World Journal of Emergency Surgery, v. 12, n. 1, p. 27.

Lin, Heng-Fu; Chen, Ying-Da; Chen, Shyr-Chyr. (2018). Value of diagnostic and therapeutic laparoscopy for patients with blunt abdominal trauma: A 10-year medical center experience. PloS one, v. 13, n. 2, p. e0193379.

Matsevych, Yevhenovych; Koto, Modise Zacharia; Aldous, Colleen. (2018). Laparoscopic-assisted approach for penetrating abdominal trauma: A solution for multiple bowel injuries. International Journal of Surgery, v. 44, p. 94-98.

Parajuli, Purushottam et al. (2018). Role of laparoscopy in patients with abdominal trauma at level-I trauma center. Surgical laparoscopy, endoscopy \& percutaneous techniques, v. 28 , n. 1 , p. $20-25$.

\section{How to cite this article (APA format):}

Magalhães, Bráulio Filgueira; Leite, Pedro de Sousa; Maia Filho, Pedro Hugo Bezerra; Sá, George Wallisson Severo de; Mascarenhas, Whallyson Pinheiro; Maia, Lucas Benevides; Lima, Ticiane Ponciano de Oliveira; Marques, Igor de Morais; Rolim, Francisco Felippe de Araújo (2019). Laparoscopy and Penetrating Abdominal Trauma: A Systematic Review. Am. In. Mult. J., October a December. (7) 4, 217-231.

Received: $11 / 25 / 2019$

Accepted: 12/11/2019 